\section{Resultados perinatológicos del servicio de Cardiopatía y Embarazo del Hospital Ginecobstétrico "Ramón González Coro" en el bienio 2000-2001, Habana}

\section{Perinatologic results of Cardiopathy and Pregnancy's Service in 2000-2001, in "Ramón González Coro" Hospital, Havana}

Roberto Viñas Oliva 1 Niobis Sánchez Ramírez 1 Pedro Román Rubio 2 Goliat Reina Gómez 3 José Oliva 4

1-4 Hospital Ginecobstétrico Ramón González Coro. Calle 21 entre 4 y 6 Municipio Plaza de la Revolución Ciudad de La Habana. Teléfono: 55 2611-13 E-mail: hgcoro@infomed.sld.cu

\begin{abstract}
Objectives: to establish the basis for treatment and care of cardiopath women in fertile age, following the criteria of evidence based Medicine, through the clinical and perinatal assessment of a group of pregnant women admitted in our service.

Methods: the women were classified according to their cardiopathy and degree of functional impairment related to the complications described during pregnancy, delivery and puerperal stage and perinatal results. The variables analysed were : type of cardiopathy and functional classification of the women, obstetrical and/or cardiovascular complications, pregnancy age at delivery, type of delivery, weigth and Apgar ponctuation in the newly born and mother and infant mortality rates.

Results: 129 acquired valvulopathies, 79 congenital valvulopathies, 18 cases of rithm disturbances. The most frequent lesions were mitral valvulopathies (92) and interauricular communications (31). 21 Patients had their condition aggravated during pregnancy. Cardiovascular complications appeared in 19 patients and obstetric ones in 142. Fourteen had to be admitted in Intensive Care Units and three died.

Concusions: these results provide an idea of development of this line of work and its impact on reproductive health. The association between functional classifications and cardiovascular complications during pregnancy, delivery, and puerperium were confirmed. Obstetrical complications were the same as in healthy patients and more than half of the devlieries were eutoccic.
\end{abstract}

Key words Maternal mortality, Prenatal care, Infant mortality, Pregnancy complications, cardiovascular

\section{Resumo}

Objetivos: establecer las bases para el tratamiento y atención de la mujer portadora de cardiopatía en edad fértil, caracterizando al grupo de gestantes atendidas en servicio.

Métodos: realizó un estudio descriptivo y las mujeres fueran clasificadas según cardiopatía y grado de afectación funcional en relación con los resultados, se decribiendo las complicaciones durante el embarazo, parto y puerperio y los resultados perinatales. Variables analizadas: tipo de cardiopatía y clasificación funcional de las mismas, complicaciones obstétricas y/o cardiovasculares, edad gestacional al parto, modo de nacimiento, peso y puntaje de Apgar en el recién nacido y mortalidad materna y del recién nacido.

Resultados: encontramos 129 valvulopatías adquiridas, 79 congénitas, 18 casos de trastornos del ritmo. Lesiones más frecuentes valvulopatías mitrales (92) y comunicaciones interauriculares (31). 21 pacientes presentaron empeoramiento funcional durante el embarazo. Aparecieron complicaciones cardiovasculares en 19 pacientes y obstétricas en 142. 14 requirieron ingreso en Servicios de Terapia Intensiva y tres fallecieron.

Conclusiones: Se confirmó la asociación entre clasificación funcional y complicaciones cardiovasculares durante el embarazo, parto y puerperio. Las complicaciones obstétricas se comportan igual que en las gestaciones de pacientes sanas y más de la mitad de los partos fueron eutócicos.

Palavras-chave Mortalidad materna, Atencion prenatal, Complicaciones cardiovasculares del embarazo, Mortalidad infantil 
Introducción

Actualmente se reconoce que la gestación puede coexistir con una cardiopatía y, sin ignorar que constituye la primera causa de morbimortalidad materna no obstétrica, ${ }^{1}$ es posible lograr un embarazo con éxito si existe una asesoría adecuada al valorar los riesgos, incluyendo los de carácter biológico, sicológicos y socioeconómicos, así como los riesgos fetales y la teratogenicidad de los tratamientos propuestos, como es el caso del uso de anticoagulantes y determina acciones específicas en materia anticoncepcional de carácter transitorio o permanente, con un uso racional de los métodos anticonceptivos, resulta fundamental para la salud reproductiva de las cardiópatas. $^{2}$

Esta evaluación permite definir si resulta aceptable la gestación en la paciente y debe incluir un análisis de los recursos que se necesitan para cubrir sus necesidades, respetando los criterios de la medicina basada en la evidencia ${ }^{3}$ y utilizando un enfoque multidisciplinario.

Las actividades se ejecutan en la atención primaria, secundaria y terciaria, teniendo como principio básico que es preferible hacer el diagnóstico y tratamiento de las cardiopatías antes del embarazo.

En la atención primaria, en la etapa preconcepcional, se realiza la prevención y tratamiento adecuado de la carditis reumática en edades pediátricas, el diagnóstico y tratamiento oportuno de las cardiopatías congénitas en niños y niñas y se intenta evaluar como mínimo tres veces al año a las mujeres con cardiopatía, priorizando a las que se encuentran en edad fértil. En esta evaluación participan los especialistas en ginecología y obstetricia y en medicina interna de los grupos básicos de trabajo en las áreas de salud, realizándose interconsultas con cardiología y, en el caso de las cardiopatías congénitas, con la consulta municipal de genética, orientándose evaluaciones periódicas en la consulta de planificación familiar y remisiones a las consultas de asesoramiento preconcepcional y de patología obstétrica de los hospitales.

La atención secundaria se establece a partir de la consulta de asesoramiento preconcepcional en los hospitales provinciales (o en más de un hospital, si las condiciones de la provincia lo permiten), integrada por cardiólogos, obstetras y psicólogos, que brindan a la pareja toda la información sobre los riesgos inherentes a su patología, según la clasificación de Clark, 4,5 posibilidades de embarazo, riesgos para el mismo y posibilidades terapéuticas previas a la gestación o durante la misma, orientando métodos anticonceptivos transitorios o permanentes de acuer- do al riesgo y los deseos de la pareja. Durante el asesoramiento debe quedar bien establecido que, sí a pesar de existir una contraindicación para el embarazo, la pareja mantiene el deseo de tener descendencia, recibirán todo el apoyo del equipo de salud, orientándose la concurrencia temprana a la consulta. Se evaluará la suspensión o cambio de medicamentos que conlleven riesgo de malformación fetal antes de iniciarse el embarazo y se solicitará asesoramiento genético cuando la mujer sea portadora de una cardiopatía congénita.

A partir del establecimiento del esquema de atención a las cardiópatas, se establece la consulta de cardiopatía y embarazo en el hospital ginecobstétrico "Ramón González Coro", centro de referencia nacional, garantizando la atención obstétrica, clínica y cardiológica de las pacientes con personal especializado en las especialidades de cardiología, ginecoobstetricia, terapia intensiva y anestesiología y los recursos mínimos para esta atención. Las pacientes se mantienen agrupadas durante el ingreso en una sala, siendo proporcional el número de camas al estimado de cardiopatías que deben ser atendidas. Las pacientes clasificadas como funcional grado I pueden ser atendidas en otros hospitales después de su evaluación.

Existe además una consulta de referencia de cardiopatía y embarazo en el "Instituto de Cardiología y Cirugía Cardiovascular", nivel terciario, donde se realiza la evaluación de las mismas, determinándose su estado funcional y las exigencias necesarias para lograr una gestación con resultados favorables, incluyendo la orientación sobre el centro hospitalario donde deberán recibir la atención prenatal y del parto y cuales son las condiciones adecuadas para la terminación del embarazo. No todas las pacientes con enfermedad cardiovascular son conocidas antes de iniciar un embarazo. Por este motivo la valoración de embarazadas en las que se sospeche una enfermedad cardíaca debe incluir un interrogatorio cuidadoso, exploración física completa y pruebas de laboratorio no invasivas para establecer el diagnóstico y pronóstico. El grado de capacidad funcional se determina según la clasificación de la American College of Cardiology (ACA) y American Heart Association (AHM), ${ }^{6}$ que sirve como guía precisa en el pronóstico materno, ya que la morbilidad y mortalidad reportadas aumentan en proporción directa con la clase funcional específica.

La frecuencia de la atención prenatal se establece de acuerdo a la gravedad de la cardiopatía, existiendo un flujograma establecido (Figura 1), valorándose las posibles complicaciones en cada control. El incremento de los elementos de riesgo o evidencias de 
descompensación en la paciente determinan el ingreso hospitalario en el servicio de cardiopatía y embarazo y si la gravedad lo requiere, la paciente es trasladada al Instituto de Cardiología y Cirugía Cardiovascular o al Servicio de Terapia Intensiva del Hospital Clínico Quirúrgico "Hermanos Amei- jeiras". En las gestaciones no complicadas el ingreso hospitalario tiene lugar a las 39 semanas, realizándose pruebas de bienestar fetal (estudios ultrasonográficos y cardiotacografía fetal) hasta el final del embarazo.

Figura 1

Protocolo para la atención de la gestante con cardiopatía.

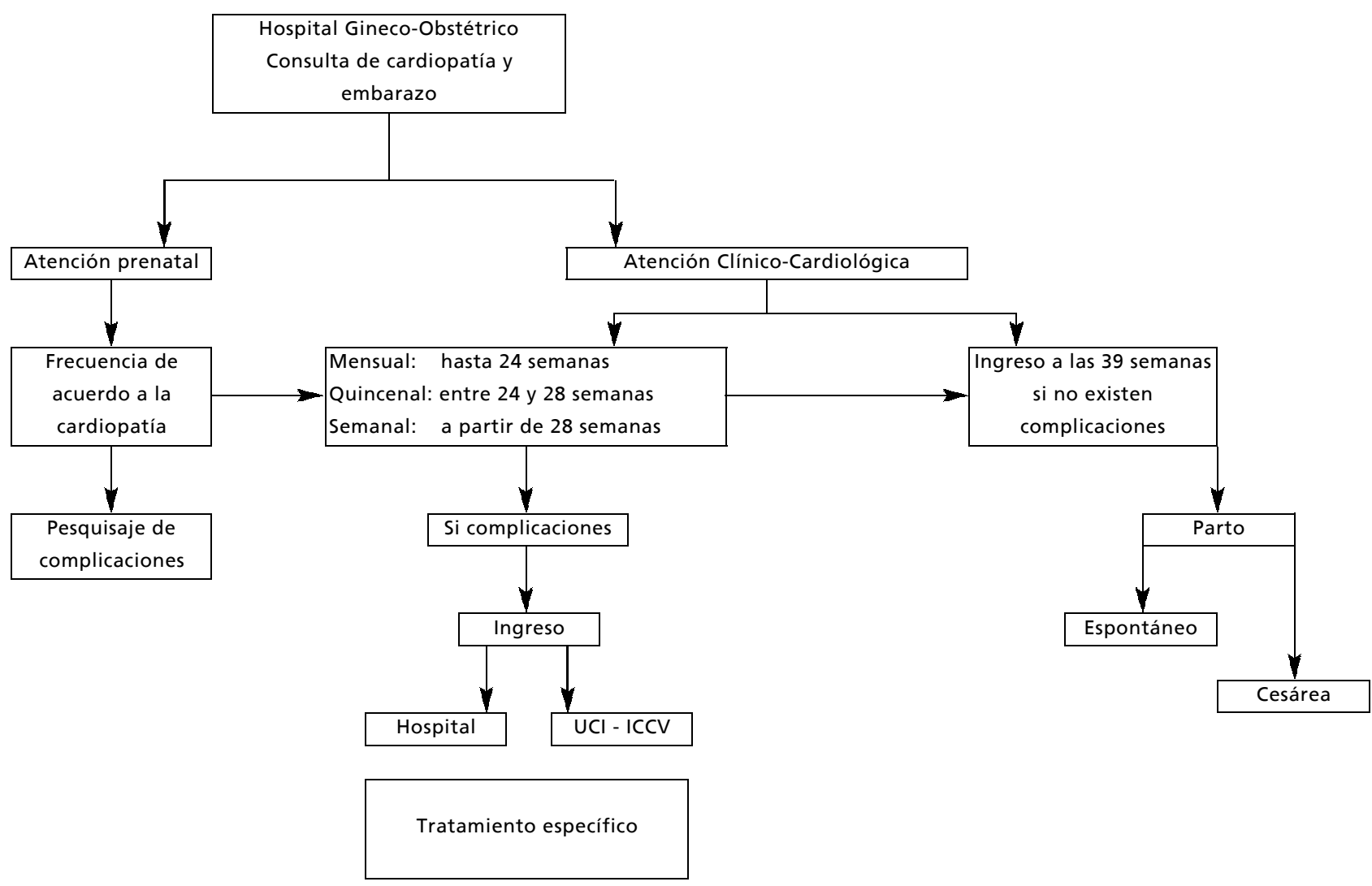

$\mathrm{UCI}=$ Unidades de Cuidados Intensivos; ICCV = Instituto de Cardiología y Cirugía Cardiovascular 


\section{Objetivo general}

Establecer las bases para el tratamiento y atención de la mujer portadora de cardiopatía en edad fértil, de acuerdo con la medicina basada en la evidencia, caracterizando desde el punto de vista clínico y perinatológico a un grupo de gestantes atendidas en nuestro servicio.

\section{Objetivos específicos}

Clasificar las gestantes según cardiopatía y grado de afectación funcional y establecer su relación con los resultados obstétricos.

Describir las complicaciones aparecidas durante el embarazo, parto y puerperio.

Describir los resultados perinatales en el grupo estudiado.

Disminuir los índices de mortalidad y morbilidad materno-infantil, originados por enfermedad cardiovascular.

\section{Métodos}

Se realizó un estudio descriptivo con elementos analíticos, observacionales, prospectivo y longitudinal del $100 \%$ de las pacientes atendidas en la consulta especializada del Servicio de Cardiopatía y Embarazo desde noviembre de 1998 hasta diciembre del 2000 , incluyendo los casos ya diagnosticados, independientemente del momento en que éste había sido realizado (previo o posterior al embarazo), clasificándose por patologías, según su clase funcional, paridad y edad al inicio de la gestación y en el momento del parto, tipo de parto, aparición de complicaciones, ya fueran estas cardiovasculares u obstétricas, resultados perinatales (incluyendo el peso al nacer y conteo de Apgar al minuto), así como resultados en mortalidad materna y perinatal en las pacientes estudiadas.

En la atención obstétrica se realizó mensuración de la altura uterina, curva de peso y determinación de frecuencia cardíaca fetal, con examen ultrasonográfico entre las 20 y 24 semanas, incluyendo ecocardiografía fetal, pudiendo realizarse otros estudios según los hallazgos del examen obstétrico.

Se realiza además examen físico general, buscando especialmente signos y síntomas de descompensación cardiovascular. A las gestantes se les realizó ecocardiograma y electrocardiograma según criterio del cardiólogo.
La conducta a seguir en relación con la terminación del embarazo fue decidida por el conjunto de facultativos, clasificándose durante el trabajo de parto en el nivel tres de atención, con vigilancia estricta y tomando medidas específicas para cada cardiopatía en particular.

\section{Resultados}

Los resultados se exponen en las tablas. De manera general podemos decir que en un total de 233 ingresos encontramos un total de 129 valvulopatías adquiridas, 79 cardiopatías congénitas, 18 casos de trastornos del ritmo cardíaco y otras cardiopatías en número de siete. (Tabla 1).

La distribución de pacientes según la clasificación funcional, utilizando los criterios de la American College of Cardiology y American Heart Association, 7 dio como resultado que 216 pacientes correspondían el inicio de su atención a la categoría Grado I, 13 a la categoría Grado II y 4 a la categoría Grado III (Tabla 2).

La distribución de las pacientes según la edad gestacional al inicio de la atención en nuestro servicio se recoge en la Tabla 3.

De acuerdo con la historia obstétrica 128 de las pacientes no tenían partos anteriores, 87 tenían un parto anterior, 16 tenían dos partos previos y dos enfrentarían un embarazo al menos por cuarta vez (Tabla 4).

La aparición de complicaciones cardiovasculares, insuficiencia cardiaca congestiva, edema agudo del pulmón, tromboembolismo pulmonar, arritmias, aparece en la Tabla 5.

Las complicaciones detectadas en el embarazo, anemia, rotura prematura de membranas, hipertensión gestacional, crecimiento intrauterino retardado, amenaza de parto pretérmino, gestorragia, pueden apreciarse en la Tabla 6.

El momento del parto aparece recogido en la Tabla 7 y su distribución según el tipo en la Tabla 8 . La inducción del parto en nuestras pacientes fue motivada por: rotura prematura de membranas, embarazo prolongado, hipertensión, crecimiento intrauterino retardado y otras causas (Tabla 9).

La indicación de cesárea estuvo determinada por las siguientes causas: sufrimiento fetal agudo, presentación pelviana, desproporción cefalopélvica, por indicación del cardiólogo, por fallo en la inducción, por cesárea anterior y por otras causas 16 (Tabla 10). 
Tabla 1

Clasificación según cardiopatía.

\begin{tabular}{|c|c|c|c|}
\hline Tipo de cardopatía & Patología & $\mathbf{n}$ & $\%$ \\
\hline \multirow[t]{10}{*}{ Valvulopatías } & Prolapso de la válvula mitral & 55 & 42,7 \\
\hline & Estenosis mitral & 20 & 15,5 \\
\hline & Insuficiencia mitral & 17 & 13,2 \\
\hline & Estenosis aórtica & 17 & 13,2 \\
\hline & Prótesis mecánica & 6 & 4,7 \\
\hline & Estenosis pulmonar & 6 & 4,7 \\
\hline & Doble lesión mitral & 4 & 3,1 \\
\hline & Doble lesión aórtica & 2 & 1,5 \\
\hline & Insuficiencia tricuspidea & 1 & 0,75 \\
\hline & Fibrosis sigmoidea aórtica & 1 & 0,75 \\
\hline Total & & 129 & 100,0 \\
\hline \multirow[t]{7}{*}{ Cardiopatías congénitas } & Comunicación interventricular & 31 & 39,2 \\
\hline & Comunicación interauricular & 23 & 29,1 \\
\hline & Tetralogía de Fallot & 10 & 12,7 \\
\hline & Coartación de la aorta & 4 & 5,0 \\
\hline & Persistencia del conducto arterioso & 3 & 3,8 \\
\hline & Estenosis pulmonar & 2 & 2,5 \\
\hline & Otras & 2 & 2,6 \\
\hline Total & & 79 & 100,0 \\
\hline \multirow[t]{2}{*}{ Trastornos del ritmo } & Bloqueo AV & 4 & 22,2 \\
\hline & Taquiarritmias & 14 & 77,8 \\
\hline Total & & 28 & 100,0 \\
\hline \multirow[t]{6}{*}{ Otras cardiopatías } & Miocardiopatía dilatada & 2 & 28,7 \\
\hline & Miocardiopatía puerperal & 1 & 14,3 \\
\hline & Miocardiopatía hipertrófica & 1 & 14,3 \\
\hline & Cardiopatía isquémica & 1 & 14,3 \\
\hline & Fibroelastosis endocárdica & 1 & 14,3 \\
\hline & Hipertensión pulmonar secundaria & 1 & 14,3 \\
\hline Total & & 7 & 100,0 \\
\hline Total & & 233 & 100,0 \\
\hline
\end{tabular}

Fuente: Historias clínicas del Servicio de Cardiopatía y Embarazo del Hospital Ginecobstétrico "Ramón González Coro". Habana, Cuba. 
Tabla 2

Estado funcional durante la gestación.

\begin{tabular}{lrrrr}
\hline & \multicolumn{2}{c}{ Al inicio de la gestación } & \multicolumn{2}{c}{ Al final de la gestación } \\
\cline { 2 - 5 } Clasificación & $\mathrm{n}$ & $\%$ & $\mathrm{n}$ & $\%$ \\
\hline Grado I & 216 & 92,7 & 212 & 91,0 \\
Grado II & 13 & 5,5 & 10 & 4,2 \\
Grado III & 4 & 1,8 & 1 & 0,6 \\
Grado IV & 0 & 0 & 233 & 100,0 \\
Total & 233 & 100,0 & & \multirow{2}{*}{. } \\
\hline
\end{tabular}

Fuente: Historias clínicas del Servicio de Cardiopatía y Embarazo del Hospital Ginecobstétrico "Ramón González Coro". Habana, Cuba.

Tabla 3

Edad gestacional al inicio de la atención.

\begin{tabular}{lcr}
\hline Edad gestacional & $\mathbf{n}$ & $\%$ \\
\hline Hasta $12 \mathrm{~s}$ & 4 & 1,7 \\
De 13 a $20 \mathrm{~s}$ & 38 & 16,3 \\
De 21 a $28 \mathrm{~s}$ & 47 & 20,2 \\
De 29 a $36 \mathrm{~s}$ & 41 & 17,6 \\
+ de $36 \mathrm{~s}$ & 10 & 4,2 \\
No captadas & 93 & 40,0 \\
Total & 233 & 100,0 \\
\hline
\end{tabular}

Fuente: Historias clínicas del Servicio de Cardiopatía y Embarazo del Hospital Ginecobstétrico "Ramón González Coro". Habana, Cuba.

Tabla 4

Distribución de pacientes según paridad.

Antecedentes obstétricos.

\begin{tabular}{lrr}
\hline Gestaciones previas & $\mathbf{n}$ & $\%$ \\
\hline Ningún parto & 128 & 55,0 \\
Uno parto anterior & 87 & 37,0 \\
Dois partos anteriores & 16 & 7,0 \\
Tres ó más partos anteriores & 2 & 1,0 \\
Total & 233 & 100,0 \\
\hline
\end{tabular}

Fuente: Historias clínicas del Servicio de Cardiopatía y Embarazo del Hospital Ginecobstétrico "Ramón González Coro". Habana, Cuba. 
Tabla 5

Complicaciones cardiovasculares

\begin{tabular}{lcc}
\hline Afectación & $\mathbf{n}$ & $\%$ \\
\hline Insuficiencia cardíaca congestiva & 6 & 2,6 \\
Edema agudo del pulmón & 2 & 0,8 \\
Tromboembolismo pulmonar & 3 & 1,3 \\
Arritmia & 8 & 3,5 \\
Total & 19 & 8,2 \\
\hline
\end{tabular}

Fuente: Historias clínicas del Servicio de Cardiopatía y Embarazo del Hospital Ginecobstétrico "Ramón González Coro". Habana, Cuba.

\section{Tabla 6}

Complicaciones obstétricas.

\begin{tabular}{lrr}
\hline Afectación & $\mathbf{n}$ & $\%$ \\
\hline Anemia & 51 & 21,9 \\
Amenaza de parto pretérmino & 3 & 1,3 \\
Rotura prematura de membranas & 46 & 19,7 \\
Gestorragia & 2 & 0,8 \\
Crecimiento intrauterino retardado & 6 & 2,6 \\
Enfermedad hipertensiva gestacional & 20 & 8,6 \\
Otras & 14 & 6,0 \\
Total & 142 & 60,9 \\
\hline
\end{tabular}

Fuente: Historias clínicas del Servicio de Cardiopatía y Embarazo del Hospital Ginecobstétrico "Ramón González Coro". Habana, Cuba.

\section{Tabla 7}

Edad gestacional en el momento del parto.

\begin{tabular}{lrr}
\hline Edad gestacional & $\mathbf{n}$ & $\%$ \\
\hline$<28 \mathrm{~s}$ & 2 & 0,8 \\
$28-32 \mathrm{~s}$ & 3 & 1,2 \\
$33-36 \mathrm{~s}$ & 11 & 4,7 \\
$37-41 \mathrm{~s}$ & 202 & 86,5 \\
42 ó más & 15 & 6,8 \\
Total & 233 & 100,0 \\
\hline
\end{tabular}

Fuente: Historias clínicas del Servicio de Cardiopatía y Embarazo del Hospital Ginecobstétrico "Ramón González Coro". Habana, Cuba. 
Tabla 8

Forma de parto.

\begin{tabular}{lrr}
\hline Tipo de parto & $\mathbf{n}$ & \% \\
\hline Eutócico & 145 & 62,2 \\
Instrumentado & 25 & 10,8 \\
Cesárea & 63 & 27,0 \\
Total & 233 & 100,0 \\
\hline
\end{tabular}

Fuente: Historias clínicas del Servicio de Cardiopatía y Embarazo del Hospital Ginecobstétrico "Ramón González Coro". Habana, Cuba.

Tabla 9

Indicaciones de inducción del parto.

\begin{tabular}{lrr}
\hline Causa & $\mathbf{n}$ & $\%$ \\
\hline Rotura prematura de membranas & 37 & 15,9 \\
Crecimiento intrauterino retardado & 3 & 1,2 \\
Hipertensión arterial gestacional & 5 & 2,1 \\
Embarazo prolongado & 10 & 4,2 \\
Otras & 15 & 6,8 \\
Total & 70 & 30,0 \\
\hline
\end{tabular}

Fuente: Historias clínicas del Servicio de Cardiopatía y Embarazo del Hospital Ginecobstétrico "Ramón González Coro". Habana, Cuba.

\section{Tabla 10}

Indicación de cesárea.

\begin{tabular}{lrr}
\hline Causa & $\mathbf{n}$ & \% \\
\hline Sufrimiento fetal agudo & 6 & 2,65 \\
Presentación pelviana & 7 & 3,1 \\
Desproporción cefalopélvica & 8 & 3,5 \\
Cesárea anterior & 6 & 2,65 \\
Indicación por cardiología & 2 & 0,85 \\
Inducción fallida & 8 & 3,5 \\
Otras causas & 16 & 7,0 \\
Total & 53 & 22,75 \\
\hline
\end{tabular}

Fuente: Historias clínicas del Servicio de Cardiopatía y Embarazo del Hospital Ginecobstétrico "Ramón González Coro". Habana, Cuba. 
Las Tablas 11 e 12 se refieren a los recién nacidos obtenidos en cuanto a distribución por puntaje de Apgar al primer minuto y peso al nacer.

Las complicaciones en el parto y/o puerperio, de causa obstétrica o cardiovascular aparecieron en un total de $20,55 \%$ de las pacientes (Tabla 13 ).
Los casos cuya gravedad determinó su traslado a Unidad de Cuidados Intensivos fueron un total de 14 (Tabla 14).

En total se produjeron tres defunciones maternas y seis defunciones perinatales (Tabla 15)

Tabla 11

Puntaje de Apgar al minuto de nacido.

\begin{tabular}{lrr}
\hline Apgar & $\mathbf{n}$ & $\%$ \\
\hline $0-3$ & 5 & 2,15 \\
$4-6$ & 3 & 1,25 \\
$>7$ & 225 & 96,6 \\
Total & 233 & 100,0 \\
\hline
\end{tabular}

Fuente: Historias clínicas del Servicio de Cardiopatía y Embarazo del Hospital Ginecobstétrico "Ramón González Coro". Habana, Cuba.

Tabla 12

Distribución por peso de los recién nacidos.

\begin{tabular}{lrr}
\hline Peso & n & $\%$ \\
\hline$<1.500 \mathrm{~g}$ & \multicolumn{1}{c}{$\%$} \\
$1.500-2.500 \mathrm{~g}$ & 20 & 1,7 \\
$2.501-3.999 \mathrm{~g}$ & 190 & 8,5 \\
$>4.000 \mathrm{~g}$ & 19 & 81,7 \\
Total & 233 & 100,1 \\
\hline
\end{tabular}

Fuente: Historias clínicas del Servicio de Cardiopatía y Embarazo del Hospital Ginecobstétrico "Ramón González

Coro". Habana, Cuba.

Tabla 13

Complicaciones del parto y el puerperio.

\begin{tabular}{lccr}
\hline Peso & Obstétricas (\%) & Cardiovasculares (\%) & Total \\
\hline Parto & 5,50 & 0,85 & 6,35 \\
Puerperio & 13,0 & 1,20 & 14,20 \\
Total & 18,5 & 2,05 & 20,55 \\
\hline
\end{tabular}

Fuente: Historias clínicas del Servicio de Cardiopatía y Embarazo del Hospital Ginecobstétrico "Ramón González Coro". Habana, Cuba. 
Ingresos en Unidad de Cuidados Intensivos.

\begin{tabular}{lcc}
\hline Causa de ingreso & $\mathbf{n}$ & $\%$ \\
\hline Insuficiencia cardíaca & 3 & 1,25 \\
Arritmia cardíaca & 5 & 2,15 \\
Edema agudo del pulmón & 1 & 0,43 \\
Tromboembolismo pulmonar & 2 & 0,85 \\
Otras & 3 & 1,25 \\
Total & 14 & 5,9 \\
\hline
\end{tabular}

Fuente: Historias clínicas del Servicio de Cardiopatía y Embarazo del Hospital Ginecobstétrico Ramón González Coro, Habana, Cuba.

Tabla 15

Resultados perinatales.

\begin{tabular}{llr}
\hline Defunciones & $\mathbf{n}$ & \% \\
\hline Maternas & 3 & 1,25 \\
Perinatales & 6 & 2,5 \\
\hline
\end{tabular}

Fuente: Historias clínicas del Servicio de Cardiopatía y Embarazo del Hospital Ginecobstétrico Ramón González Coro, Habana, Cuba.

\section{Discusión}

Según se aprecia en la Tabla 1, las cardiopatías más frecuentes en nuestras pacientes fueron las lesiones valvulares, y dentro de estas el prolapso de la válvula mitral, la estenosis mitral, la insuficiencia mitral y la estenosis aórtica. El prolapso de la válvula mitral es una patología frecuente, tanto en su forma congénita o en la adquirida por fiebre reumática y cursa con gran frecuencia de modo asintomático, no representando ningún riesgo para la gestación en gran parte de los casos, pero además es frecuente el sobrediagnóstico de esta valvulopatía.

Dentro de las cardiopatías congénitas los hallazgos más frecuentes fueron: comunicación interauricular, comunicación interventricular, tetralogía de Fallot, estenosis aórtica y coartación de la aorta. Pese a que la incidencia de cardiopatía congénita en el recién nacido es de $4-5 \%$ por 1.000 nacidos vivos (elevándose a $4 \%$ en las madres afectadas por cardiopatía congénita), 7,8 en nuestro estudio solo se constató un recién nacido portador de enfermedad cardíaca congénita incompatible con la vida, lo que confirma la presunción de que estas afecciones son producto de la interacción genética con factores ambientales, con la posible influencia de la hipoxia, los medicamentos y las carencias nutricionales. 9 La real incidencia de este problema la desconocemos, ya que no se pudo efectuar el seguimiento en el primer año de vida de todos los niños.

Los trastornos del ritmo predominantes fueron las taquiarritmias, $77 \%$ del total de las mismas, que aparecieron en el $6 \%$ del total de pacientes estudiadas y constituyen un factor de agravamiento al afectar el estado hemodinámico de las pacientes. 10

Pese a que al inicio de la gestación 216 pacientes correspondían a la clasificación funcional I, 13 a la categoría II y quatro a la categoría III, al final de ésta 10 pacientes habían pasado a la categoría III y un a la categoría IV, lo que significa un empeoramiento del estado de salud en, al menos, $3 \%$ de las pacientes, cifra relativamente baja y acorde con lo esperado de acuerdo con el estado inicial de nuestras pacientes. A duas de las pacientes inicialmente clasi- 
ficadas como grado III se les realizó en el tercer trimestre de la gestación valvuloplastia percutánea mitral por balón, mejorando las variables clínicas y hemodinámicas de las pacientes, que pasaron a la clasificación I.

La mayor parte de las pacientes eran procedentes de otros centros y no fueron captadas inicialmente en nuestro servicio. En muchos casos el diagnóstico de cardiopatía fue hecho durante el embarazo y en ocasiones, muy tardíamente.

El 55\% de nuestras pacientes eran primigestas. Este es un elemento importante dentro de los criterios para apoyar o desaconsejar una gestación en pacientes de alto riesgo. El 37\% tenía un embarazo anterior y solo el $8 \%$ tenían dos o más hijos vivos, lo que realmente constituye una contradicción y nos permite asegurar que la labor educativa con estas pacientes ha sido deficiente, pues no han sido capaces de realizar una adecuada valoración riesgo/beneficio que las indujera a recurrir a una adecuada planificación familiar.

La aparición de complicaciones cardiovasculares constituye el $8,2 \%$ del total, siendo las más frecuentes las arritmias 11 y la insuficiencia cardíaca congestiva.

Los partos a término constituyeron un $86,5 \%$, en tanto que los pretérmino y postérmino tuvieron cifras similares. Estas cifras son muy similares a las descritas en la literatura y están correlacionadas con la gravedad de la cardiopatía, la limitación que esta produce y el grado de hipoxia crónica.

En relación con la vía del parto predominó el parto fisiológico, sin que se produjeran complicaciones en el período expulsivo. En su mayoría corresponden a pacientes con clasificación funcional I, pero no existe correlación estadísticamente significativa entre el modo de nacimiento y la clasificación funcional.12 Las cesáreas se presentan con mayor frecuencia como indicación por situaciones obstétricas, aunque en pacientes con cardiopatías cianóticas severas 13 se trata de evitar el esfuerzo del parto, al igual que en algunas miocardiopatías ${ }^{14}$ y aortitis.

Algunos autores sugieren que la cesárea es preferible al parto vaginal en situaciones como la coartación aórtica, el síndrome de Marfán y las aortitis, por el riesgo de disección aórtica, y en las cardiopatías cianóticas severas, en que el trabajo de parto aumenta el shunt de derecha a izquierda, agravando la hipoxemia fetal.15,16

Del total de recién nacidos vivos (228), 3\% obtuvieron Apgar inferiores a 7; tres de ellos en madres clasificadas como grado II, dos como grado I y uno como grado III, indicando que no existe una relación directa con la enfermedad materna, sino con las propias condiciones del feto y el modo de nacimiento, cesárea y uso de anestesia general.17,18

En el 7,26\% de los nacimientos los recién nacidos presentaron un peso inferior a 2.500 gramos, distribuidos proporcionalmente entre pacientes con clasificación funcional I y II, llamando la atención que, contrariamente a lo descrito en la literatura, no existe bajo peso dentro de las pacientes clasificadas como grado funcional III. 19

Las cifras de mortalidad perinatal revelan una incidencia de $2,5 \%$. Hay autores que describen cifras de hasta $18 \%$ y otros, en series más pequeñas que la nuestra, no refieren mortalidad. Es indiscutible que la viabilidad fetal está determinada por la clasificación funcional, cianosis y uso de anticoagulante 20 en la madre, pues en nuestro estudio correspondieron con tres pacientes con prótesis valvulares que requerían tratamiento anticoagulante, una era portadora de estenosis mitral y sufrió un shock anafiláctico durante la gestación, otra era una hipertensa crónica con arritmia ventricular compleja y la última tenía antecedentes de estenosis pulmonar.

La explicación fisiopatológica de la mortalidad perinatal se encuentra relacionada con la insuficiente irrigación útero-placentaria y la hipoxemia, con la consiguiente disminución del aporte de oxígeno y nutrientes al feto. 21

Los cambios fisiológicos en el embarazo significan un desafío a la reserva funcional del sistema cardiocirculatorio, lo que se manifiesta en grados variables de insuficiencia cardíaca congestiva (ICC), edema agudo del pulmón (EAP) y eventualmente la muerte.

La mortalidad materna tuvo una incidencia de $1,2 \%$, con un total de tres defunciones. Se reporta una estenosis aórtica clasificada como ligera grado I, con diagnóstico anatomopatológico de estenosis severa, la cual presentó un cuadro de EAP irreversible en el curso de una cesárea iterada; otro caso corresponde con diagnóstico de estenosis mitral severa con grado funcional III que presentó cuadro de EAP irreversible durante el parto, a la que se realizócomisurotomía mitral abierta a las 72 horas, falleciendo en el postoperatorio y el tercer caso era una portadora de prótesis mitral con grado funcional II a la que una neumopatía inflamatoria llevó a la muerte por insuficiencia respiratoria aguda. Como describen algunos autores la cardiopatía empeora un grado de la clasificación funcional durante la gestación.22

En conclusion podremos decir que predominaron las valvulopatías reumáticas como causa de cardiopatía; el grado funcional más frecuente resultó ser el tipo I (más de las $2 / 3$ partes de las pacientes estudiadas); no se confirmó repercusión significativa en 
los recién nacidos; se confirmó asociación entre clasificación funcional y complicaciones cardiovasculares durante el embarazo, parto y puerperio; las complicaciones obstétricas se comportaran igual que en las gestaciones de pacientes sanas y más de la mitad de los partos fueron eutócicos.

\section{Referencias}

1. Elkayam U. Pregnancy and cardiovascular disease. In: Braunwald E, editor. Heart disease. a textbook of cardiovascular medicine. 5. ed. Philadelphia: WB Saunders; 1997. p. 1843-64.

2. McAnuklty J, Metcalfe J, Ueland K. Cardiopatías y embarazo. In: Hurts WJ, editor. El corazón. La Habana: Ed. Revolucionarias; 1981.v.1, p. 1772-4.

3. Oakley CM. Pregnancy and heart disease. In: Yusuf S, Cairns JA, Camm AJ, Fallen EL, Gersh B., editors. Evidence based cardiology. 11. ed. London: BMJ; 1998. p. 91535.

4. Clark SL, Cotton DB, Lee W. Central hemodinamics assesment of normal term pregnancy. Am J Obstet Ginecol 1989; 161: 1439-42.

5. American College of Obstetrician and Ginecologist. Cardiac disease in pregnancy. Tec Bull 1992 (168).

6. ACA (American College of Cardiology), AHM (American Heart Association). Guidelines for the management of patients with valvular heart desease. J Am Coll Cardiol 1998; 32: 1486-588.

7. Gravitz RG, Joffres MR, Collins-Nakai RL. Congenital heart disease: incidence in the first year of life. Am Epidemiol 1988; 128: 381-8.

8. Perloff JK, Child JS. Congenital heart disease in adults. Pregnancy and congenital heart disease: the mother and the fetus. 2. ed. Filadelphia: WB Saunder; 1998. p. 144 64.

9. Lougheed J. Maternal nutrition and the development of congenital heart disease: is there a link to folic acid Front Fetal Heart 2001; 3: 204-5.

10. Wolbrette D, Patel H. Arrhythmias and women. Curr Opin Cardiol 1999; 14: 36-43.

11. Joglar JA, Page RL. Treatment of cardiac arrhytmias during pregnancy: safety considerations. the University of Texas Southwestern Medical Center, Dallas, USA. Drug Saf 1999; 20: 85- 94.

12. Arias F. Cardiopatía y embarazo. In: Arias F. Guía práctica para el embarazo y el parto de alto riesgo. 3. ed. Madrid: Mosby-Doyma; 1995. p. 217-33.
13. Siu S, Chitayat D, Webb G. Pregnancy in women with congenital heart defects: what are the risks? [editorial]. Heart 1999; 81: 225-6.

14. Villanueva González MA, Cabral Castaneda F, Ruiz Anguas J, Martínez Moreno F, Villagrana Zesati JR, Ahued Ahued JR. Miocardiopatía dilatada y embarazo: informe de tres casos y revisión de la literatura. Ginecol Obstet Mex 1999; 67: 173-5.

15. Oakley C. Management of labor and delivery in the high risk patient. In: Oakley C, Elder M, editors. Heart disease in pregnancy. London: BMJ; 1997. p. 375-9.

16. Zuber M, Gautschi N, Oechslin E, Widmer V, Kiowski W, Jenni R. Outcome of pregnancy in women with congenital shunt lesions. Heart 1999; 81: 271-5.

17. Autore C, Brauneis S, Apponi F, Commisso C, Pinto G, Fedele F. Epidural anesthesia for cesarean section in patients with hypertrophic cardiomyopathy: a report of three cases. Anesthesiology 1999; 90: 1205-7.

18. Paix B, Cyna A, Belperio P, Simmons S. Epidural analgesia for labour and delivery in parturient with congenital hypertrophic obstructive cardiomyopathy. Anaesth Intensive Care 1999; 27: 59-62.

19. Enfermedades cardiovasculares. In: Williams Obstetrics: Complicaciones médicas y quirúrgicas del embarazo. 20. ed. Madrid: Médica Panamericana; 2001. p. 1003-25.

20. Vitale N, De Feo M, De Santo LS, Polloce A, Tedesco N, Cotrufo M. Dose-dependent fetal complications of warfarin in pregnant women with mechanical heart valves. J Am Coll Cardiol 1999; 33: 1637- 45.

21. Horak MJ, Nadel ES, Brown DF. Dyspnea in pregnancy. J Emerg Med 1999; 17: 507-11.

22. Birincioglu CL, Kucuker SA, Yapar EG, Yildiz U, Ulus AT, Yamak B Katircioglu-SF; Tasdemir-O. Perinatal mitral valve intervencions: a report of 10 cases. Ann Thorac Surg 1999; 67: 1312-4.

Recebido em 3 de junho de 2002

Versão final reapresentada em 25 de outubro de 2002

Aprovado em 17 de novembro de 2002 